\title{
Control of droplet movement on an inclined wall with sawtoothed wettability pattern by applying ultrasonic vibration
}

Kenji Katoh, Hiroki Tamura, Eriko Sato, Tatsuro Wakimoto

\begin{tabular}{|c|c|}
\hline Citation & Experiments in Fluids, 59(9); 141 \\
\hline Issue Date & 2018-09 \\
\hline Type & Journal Article \\
\hline Textversion & author \\
\hline $\begin{array}{l}\text { Electronic } \\
\text { supplementary } \\
\text { material }\end{array}$ & $\begin{array}{l}\text { Electronic supplementary material is available at } \\
\text { https://doi.org/10.1007/s00348-018-2592-2. }\end{array}$ \\
\hline Rights & $\begin{array}{l}\text { This is a post-peer-review, pre-copyedit version of an article published in } \\
\text { Experiments in Fluids. The final authenticated version is available online at: } \\
\text { https://doi.org/10.1007/s00348-018-2592-2 }\end{array}$ \\
\hline DOI & $10.1007 / \mathrm{s} 00348-018-2592-2$ \\
\hline
\end{tabular}

\author{
Self-Archiving by Author(s) \\ Placed on: Osaka City University
}

KATOH, K., TAMURA, H., SATO, E., \& WAKIMOTO, T. (2018). Control of droplet movement on an inclined wall with sawtoothed wettability pattern by applying ultrasonic vibration. Experiments in Fluids. 59. DOI: 10.1007/s00348-018-2592-2 


\title{
Title Page
}

\section{Title:}

Control of Droplet Movement on an Inclined Wall with Saw-toothed Wettability Pattern by Applying Ultrasonic Vibration

\section{Authors:}

Kenji Katoh $^{1}$, Hiroki Tamura ${ }^{1}$, Eriko Sato ${ }^{2}$, and Tatsuro Wakimoto ${ }^{1}$

1 Department of Mechanical Engineering, Osaka City University, Sugimoto, Sumiyoshi-ku, Osaka 558-8585, Japan

2 Department of Applied Chemistry and Bioengineering, Osaka City University, Sugimoto, Sumiyoshi-ku, Osaka 558-8585, Japan

\section{Corresponding author:}

Kenji Katoh, e-mail: katoh@mech.eng.osaka-cu.ac.jp, phone: +81-6-6605-2665

orcid: 0000000006035779

\section{Acknowledgments and Funding Information}

A part of this research was supported by Japan Society for the Promotion of Science (JSPS) Grant Number $15 \mathrm{~K} 05802$.

\begin{abstract}
This study deals with the control of the movement of liquid droplets rolling down an inclined plate based on the differences in the wettability of the plate. We used a photoreactive polymer poly(7-methacryloyloxy coumarin) (PMC) whose molecular structure can be changed reversibly to realize different wettabilities by ultraviolet irradiation. We proposed employing saw-tooth patterns at boundaries between areas with different contact angles to control the droplet trajectory. Furthermore, we experimentally observed that the droplet moves along a line inclined to the direction of gravity. The droplet behavior can be analyzed using a theoretical model based on the droplet dynamics wherein the surface tension acting on the contact line and the gravitational force are considering. The theoretical results suggest that inclination from the gravitational direction can be increased if the advancing contact angle is reduced. In the experiments conducted herein, ultrasonic vibration was applied to the inclined plate to reduce the contact angle hysteresis. The results showed that the advancing contact angle actually decreased and that the droplet trajectory was controlled to realize motion along a line with inclination angle almost twice of that realized without vibration.
\end{abstract}




\section{Introduction}

Recently, lab-on-a-chip devices and microreactors using small amounts of liquid have become popular in the fields of pharmaceuticals and chemical engineering as they help realize rapid heating and cooling, highly efficient interfacial reactions, and system capacity minimization (Abgrall and Gué 2007; Gupta et al. 2017; Xi et al. 2017). These devices often employ methods that can change the wettability or surface tension locally in order to control the motion of droplets on a wall surface (Darhuber and Troian 2005).

The most popular technique to drive droplets is electrowetting-on-dielectric (EWOD) actuation. In this technique, a decrease in the contact angle of the droplet on a charged dielectric layer is employed to control the droplet motion. This technique has been widely researched to achieve efficient actuation. Lu et al. observed the deformation process of a droplet moving between two simple horizontal electrodes in a Hele-Shaw cell and simulated the process numerically ( $\mathrm{Lu}$ et al. 2007). In other studies, the continuous movement of droplets on consecutive electrodes was investigated. The droplet speeds were measured, and the optimal electrode geometries and electrical control conditions were proposed (Adbelgawad et al. 2009; Jain et al. 2017; Nahar et al. 2016; Suzuki et al. 2010). Fobel et al. (2013) developed an integrated control system for droplet actuation using EWOD. Further, research has reported that other movements, e.g., uphill climbing, jumping, and coalescence of two droplets, can also be achieved by EWOD actuation (Bhaumik et al. 2014; Datta et al. 2015; Lee et al. 2012).

Other methods to change the wettability of the surface too have been proposed, e.g., laser irradiation, which we proposed in previous research. We developed self-assembled monolayers (SAMs) that exhibit different wettablities upon laser irradiation. The droplet manipulation on the SAMs was comparable to that achieved by EWOD actuation (Katoh et al. 2010; Wakimoto et al. 2013).

The Marangoni force induced by temperature difference is also utilized for droplet actuation. In previous works, two heat sources were embedded in a wall to generate a constant temperature gradient and the velocity of a droplet driven by the Marangoni force was measured (Chen et al. 2005; Pratap et al. 2008; Zhao et al. 2011). Further, the velocity and temperature distributions of a moving droplet were analyzed via numerical simulations (Karapetsas et al. 2017).

Some researchers adopted a more direct transfer of the driving force to the droplet by applying a magnetic force to a droplet containing magnetic material (Hutama and Oleschuk 2017; Zhang and Nguyen 2017) or by applying vibrations to a wall with anisotropic structures such as oblique micro pillars (Agapov et al. 2014; Dong et al. 2017; Shastry et al. 2006; Yeo and Friend 2014).

Although the abovementioned methods enable droplet actuation, they require continuous supply of energy from external devices such as electric power sources, heat generators, and lasers. Furthermore, the methods based on EWOD and Marangoni force require the embedment of electrodes and heat sources. The insertion of these additional components nullifies the inherent advantages of reduction of size, cost, and energy consumption of the lab-on-a-chip technology. 
In the present research, we focused on droplets falling on an inclined plate under the influence of only gravity. We attempted to control the direction of droplet motion by manipulating the wettability of the plate surface. In a previous study, we considered a method of changing the direction of droplet motion falling under gravity by patterning regions with different wettabilities on the wall (Katoh et al. 2016); for this purpose, we used a photoreactive polymer poly(7-methacryloyloxy coumarin) (PMC) whose wettability can be changed by ultraviolet (UV) irradiation. A test plate with a geometrical pattern of areas with different wettabilities was created by locally irradiating wall with UV light, and droplet behavior on the inclined test plate was observed experimentally. A saw-tooth pattern of the wall surface was adopted to change the direction of droplet movement from the gravitational direction. Experimental observations showed that droplets could be made to fall at a certain tilt away from the direction of gravity. In order to theoretically determine the trajectory of the droplet on the inclined plate, the surface tension force on the droplets was estimated by considering the contact angle varying along on the triple-phase contact line. The theoretical results could approximate the actual observed trajectory efficiently.

However, to apply the proposed method to a real system, it may be necessary to control the droplet motion more effectively. In the present study, to enhance the effect of motion control through changes in wettability, we proposed a method to reduce resistance to droplet movement from the resultant surface tension caused by contact angle hysteresis. Usually, the contact angle hysteresis between the advancing and receding contact angles may be caused by the irreversible energy barrier with the movement of the contact line on a wall with roughness or impurities. Some authors suggested that the energy barrier and hence the contact angle hysteresis can be reduced by applying vibrations to the wall (Katoh et al. 2010, Volpe et al 2002). In this study, we propose a method to apply ultrasonic vibrations to a wall surface to reduce the contact angle hysteresis and achieve more effective control of droplet movement. First, we considered the influence of changes in the contact angle on the droplet motion based on the previously reported theoretical model. Then, we experimentally studied the direction of falling of the droplet under ultrasonic vibration. A step-type pattern with areas of different wettabilities was proposed, and a saw-tooth pattern was applied to realize more flexible droplet control against gravity.

\section{Experimental device and method}

\subsection{Experimental device}

A schematic of the experimental device used in this research is shown in Fig. 1. The test plate was placed horizontally. A droplet with a specific volume $V(15-30 \mu \mathrm{L})$ was dropped on the plate by using a microsyringe. Then, the test plate was tilted to the specified angle by using a worm gear. The motion of the droplet rolling down the plate surface was observed using a digital camera. Three test plates were used for each experimental condition, and three measurements were taken for each plate. Thus, a total of nine measurements were made to obtain the mean droplet motion trajectory under each experimental 
condition.

We performed measurements with and without the application of ultrasonic vibrations. In the former case, we used a bolt-clamped Langevin oscillator with a frequency of $28.1 \mathrm{kHz}$ (Fuji Ceramics FBL28452HS) as the oscillator. An alternating current of $28.1 \mathrm{kHz}$ was output from a function generator (NF Circuit Design Block manufactured, DF1906), and an amplifier (DENON, AE390) was used to amplify the voltage to drive the ultrasonic oscillator. This oscillator was not equipped with any horn for focusing the vibration. We applied a coat of epoxy gel as an acoustic couplant on the surface of the vibrator and placed the test plate on this coating. The amplitude of the wall-surface vibration was measured as $5.3 \mu \mathrm{m}$ by using a laser-displacement meter (KEYENCE, LK-G5000). The low efficiency of the oscillator $(\sim 2 \%)$ resulted in a rise in the temperature of the surface of the test plate; however, we verified that this temperature rise was negligible (within $1{ }^{\circ} \mathrm{C}$ ) during the test.

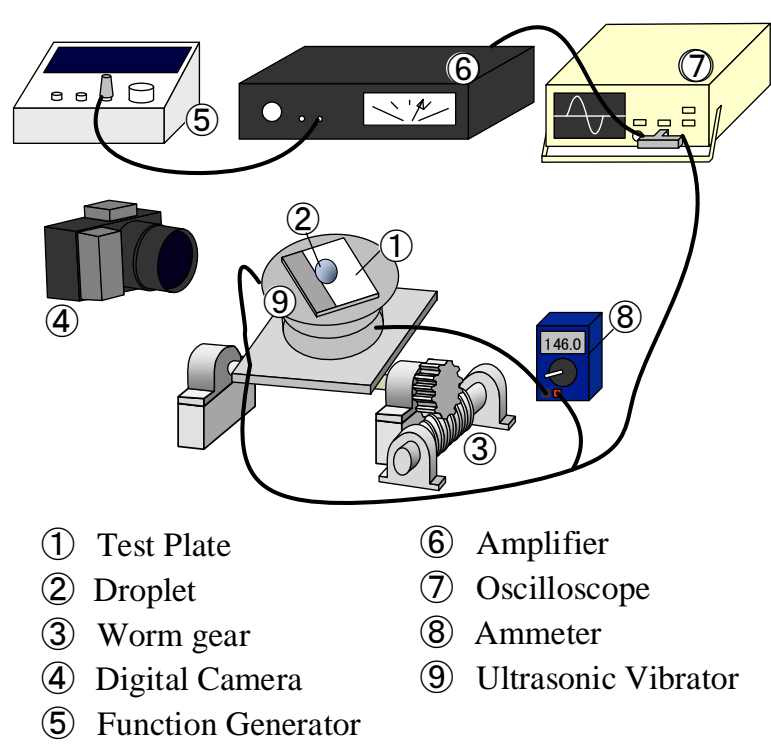

Fig. 1 Schematic of the experimental apparatus

\subsection{Test liquids, test plates, and contact angles}

In the experiments, a $20 \%$ water solution of ethanol and distilled water (used in our previous study) were used as the test liquids. Their physical characteristics are listed in Table 1, where $\rho$ represents the density and $\sigma$ represents the surface tension.

The test plates were glass boards of dimensions approximately $25 \mathrm{~mm} \times 25 \mathrm{~mm}$. The plates were spin-coated $(250 \mathrm{rpm}$ for $10 \mathrm{~s}$, followed by $2500 \mathrm{rpm}$ for $90 \mathrm{~s})$ with PMC $(0.5 \mathrm{wt} \%$ solution in 1,1,2,2-tetrachloroethane). PMC was synthesized and purified following the methods reported previously 
Table 1 Physical properties of the test liquids at $20( \pm 1)^{\circ} \mathrm{C}$

\begin{tabular}{c|c|c}
\hline \hline Test liquids & $\rho\left[\mathrm{kg} / \mathrm{m}^{3}\right]$ & $\sigma[\mathrm{mN} / \mathrm{m}]$ \\
\hline Distilled water & $998 \pm 0.2$ & $72.8 \pm 0.3$ \\
\hline Ethanol 20\% solution & $971 \pm 0.2$ & $39.5 \pm 0.3$ \\
\hline
\end{tabular}

Table 2 Contact angles (without ultrasonic vibration)

\begin{tabular}{c|c|c|c|c}
\hline \hline Test liquids & $\theta_{A}\left[{ }^{\circ}\right]$ & $\theta_{R}\left[{ }^{\circ}\right]$ & $\theta_{A}{ }^{\circ}\left[{ }^{\circ}\right]$ & $\left.\theta_{R}{ }^{\circ}{ }^{\circ}\right]$ \\
\hline Distilled water & 73.6 & 40.1 & 78.4 & 43.1 \\
\hline Ethanol $20 \%$ & 66.7 & 32.0 & 71.1 & 35.0 \\
\hline
\end{tabular}

Table 3 Contact angles (with ultrasonic vibration)

\begin{tabular}{c|c|c|c|c}
\hline \hline Test liquids & $\theta_{A}\left[{ }^{\circ}\right]$ & $\theta_{R}\left[^{\circ}\right]$ & $\theta_{A}{ }^{\prime}\left[{ }^{\circ}\right]$ & $\left.\theta_{R}{ }^{\circ}{ }^{\circ}\right]$ \\
\hline Distilled water & 65.7 & 39.7 & 67.6 & 40.9 \\
\hline Ethanol $20 \%$ & 61.9 & 31.6 & 63.8 & 32.4 \\
\hline
\end{tabular}

(Katoh et al. 2016; Sato et al. 2013). Further, [2+2] dimerization by cycloaddition occurs if a thin-membrane coumarin inducer is irradiated with UV light of wavelength greater than $300 \mathrm{~nm}$ (Morrison et al. 1966; Obi et al. 1999). This reaction is heat forbidden; hence, by irradiating a thin membrane through a photomask, a dimerized region that can be easily patterned is created. In this study, photoirradiation was carried out for $1 \mathrm{~h}$ under atmospheric conditions using an ultrahigh pressure mercury lamp (Moritex MUV-250U). The light was passed through a Pyrex glass filter to eliminate wavelengths less than $290 \mathrm{~nm}$. The spin-coated thin-membrane was placed $10 \mathrm{~cm}$ away from the light source. The light intensity was measured as $9.0 \mathrm{~mW} / \mathrm{cm}^{2}$ by a UV power meter (USHIO, UIT-101) equipped with a UVD-365PD optical receiver (330-390 nm). The surface free energy is reduced owing to the changes in the chemical structure due to dimerization, and the contact angle increased by $\sim 5^{\circ}$ (Katoh et al. 2016; Li et al. 1997; Sato et al. 2012). Moreover, if the dimers are irradiated with short-wavelength light, it is possible to recover the state of coumarin inducer and the wettability (Li et al. 1997; Sato et al. 2012).

The contact angle of the test plate with respect to the test liquids was obtained from the measurements of the shape of the axisymmetric sessile droplet on the horizontal surface (Lahoon et al. 1996). Tables 2 and 3 list the measurement results of the advancing and receding contact angles, $\theta_{A}$ and $\theta_{R}$, respectively, with respect to each liquid used; $\theta_{A}{ }^{\prime}$ and $\theta_{R}{ }^{\prime}$ are the values after irradiation with UV light. Table 2 lists the results obtained when no ultrasonic waves were applied, and Table 3 lists the results when ultrasonic 
waves were applied. The results presented in the table are the mean values for the total of 18 times that the six measurements were taken for each of the three test plates. The standard deviation was $0.7^{\circ}$. The data in Table 2 show that the increase in contact angle upon UV irradiation, $\Delta \theta$, is $\sim 3^{\circ}-5^{\circ}$. When ultrasonic vibration was applied (Table 3), the values of the receding contact angle $\theta_{R}$ were similar to those obtained with no additional vibration (Table 2). On the other hand, upon the application of ultrasonic vibrations, the advancing contact angle was reduced by $\sim 8^{\circ}$ in water and by $\sim 5^{\circ}$ in an ethanol solution; hence, the contact angle hysteresis, i.e., $\left(\theta_{A}-\theta_{R}\right)$ was also reduced. Past research suggests that the magnitude of the contact angle hysteresis $\left(\theta_{A}-\theta_{R}\right)$ is equivalent to the range of the contact angles for which many metastable states of the droplets attached to the solid surface exist. Under ultrasonic vibration, the droplet-attachment state nears the equilibrium state with the minimum energy because the energy barriers between adjacent metastable states can be overcome by vibration; hence, it is expected that the contact angle hysteresis is reduced (Katoh et al. 2010, Volpe et al 2002). The decrease in contact angle hysteresis implies a reduction in the resistance arising from the surface tension acting on the contact line when the droplet moves under an external force (Katoh et al. 2010). The data in Table 3 show that under ultrasonic vibration, the change in the contact angle $\Delta \theta$ of $\sim 2^{\circ}$ due to UV irradiation was smaller than that obtained without vibration. In conclusion, although ultrasonic vibration could reduce the resistance resulting from contact angle hysteresis, it could weaken the effect of changes in wettability caused by UV irradiation. This conflicting effect of ultrasonic vibration will be discussed further based on the theoretical model in Section 3.

In this section, we briefly describe the settings of inclination angle $\varphi$ of the test plate from the horizontal (Fig. 1). Based on the measured values of the contact angle, the critical angle $\varphi_{C}$ at which droplets fall without stopping on the test plate can be obtained from the following relationship (Katoh et al. 2016):

$$
\varphi_{C}=\sin ^{-1}\left[\frac{\sigma B\left(\cos \theta_{R}-\cos \theta_{A}^{\prime}\right)}{\rho g V}\right] .
$$

In the above equation, $B$ is the maximum width of the droplet-attaching surface, and $g$ is the gravitational acceleration. At $\varphi_{C}$, in Eq. (1), the maximum resistance due to surface tension and the gravity acting on the droplets become equal. Through this experiment, the effect of application of ultrasonic vibration on the movement of the droplets was evaluated; for each condition, the droplet volume was so selected that the critical angle was $\sim 55^{\circ}$ and the angle of inclination was set to a value greater than $\varphi_{C}$ by $\sim 0.8^{\circ}$. The droplet volume was several tens of cubic millimeters. The width $B$ in Eq. (1) can be obtained from the droplet shape on the horizontal plate as determined by the axisymmetric Laplace equation representing the force balance between surface tension and static pressure because the maximum width does not change upon tilting the test plate (Katoh et al. 2016; Katoh et al. 2006). 


\subsection{Patterning on the walls}

The surface patterns on the test plate used in this experiment are shown in Fig. 2. One part of the plate was irradiated with UV light passed through a mask to obtain regions patterned with different contact angles. Patterns A and B shown in Figs. 2(a) and 2(b) are identical to those in the previous study. Pattern $\mathrm{C}$, which is a step-type pattern, in Fig. 2(c) is newly applied in this study. The gray sections in the figures represent UV-irradiated regions with poor wettability. The angle $\mu$ shown in Patterns B and C (Fig. 2) is the target angle of incline of the droplet movement from the direction of gravity. The value of $\mu$ was set with reference to the movement of the droplet under Pattern A. The details are described in Section 4.

Figure 3 shows the expected movement of droplets falling on each test plate. In the case of Pattern A [Fig. 3(a)], if droplets enter a region with poor wettability, the resultant surface tension to the right direction is generated based on the difference between the contact angles in the two regions. Thus, the falling direction is inclined away from the direction of gravity. However, after the droplet travels some

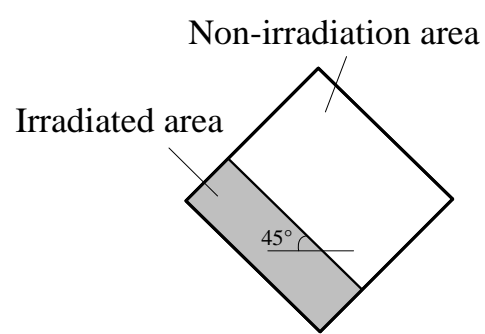

(a) Pattern A

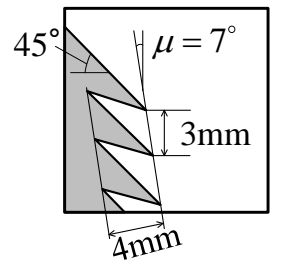

(b) Pattern B (saw tooth type)

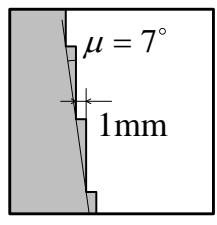

(c) Pattern C (step type)

Fig. 2 Test plates patterned with different wettabilities by irradiation of ultraviolet (UV) light (gray area indicates the area with poor wettability resulting from UV irradiation)

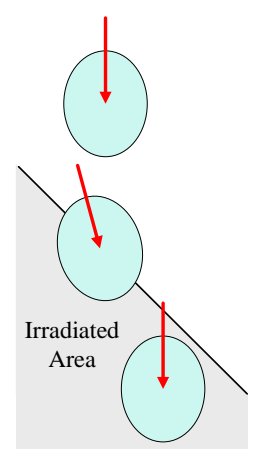

(a) Pattern A

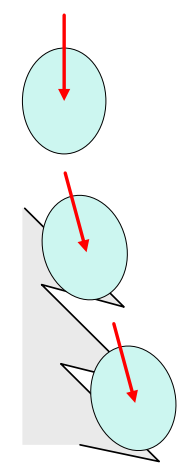

(b) Pattern B

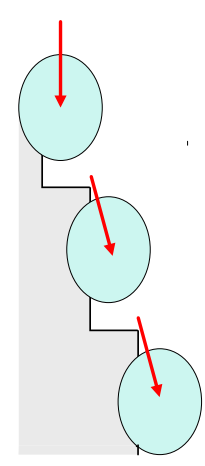

(c) Pattern C

Fig. 3 Schematic of droplet movement on each test plate when entering an area with poor wettability 
distance on the plate, the attachment surface of droplet is wholly within the region irradiated by UV light. Then, the transversal force due to the change in the contact angle disappears, and the droplet moves in the direction of gravity. In order to make the droplets fall continuously in a set direction inclined from the gravitational direction, the saw-tooth Pattern B was applied. As shown in Fig. 3(b), the droplets whose direction of motion changed after entering an irradiated area enter a non-irradiated region before moving in the direction of gravity; hence, the transversal force is reset once before re-entering the irradiated region. By repeating this action, the droplets can be made to move at a fixed incline angle from the direction of gravity (Katoh et al. 2016). Different geometrical patterns were employed in preliminary experiments to realize this effect. Based on the observed results, we chose a simple saw-tooth shape with straight lines as a promising pattern [Fig. 2(b)]. The geometrical parameters of the pattern were determined appropriately by referring to the droplet scale used in this experiment. In the step-form Pattern C [Fig. 3(c)], droplets enter the region with poor wettability and move sideways. When this displacement is saturated, they re-enter into the next stepped region with poor wettability, resulting in continuous horizontal movement. The effects of these patterns are described in Section 4.

\section{Influence of the contact angle on the direction of motion of the droplet}

We first provide an overview of the theoretical model considered in a previous report on droplet movement on a wall (Katoh et al. 2016). Then, based on the representative values of the forces acting on these droplets, we discuss the changes in the direction of motion of the droplets accompanying the changes in the contact angle.

\subsection{Theoretical model of droplet movement}

Figure 4 schematically shows the surface of the droplet in contact with the wall, hereinafter referred to as the "attached surface," when droplets enter the Pattern A region. In the figure, $x$ is the direction of gravity; $y$, the transverse direction to the plate surface; and $\beta$, the angle of incline of the direction of motion of the droplets with respect to gravity. The shape of the attached surface of the droplet is assumed oval, as shown in Fig. 4. The radii of the top and bottom arcs are denoted as $R$, with the length of the straight part as $m R$, where $m=0.6$ within the scope of this experiment. Note that $m$ can be actually estimated from our theoretical method to obtain the three-dimensional droplet profile on a tilted plate (Higashine et al. 2008). However, for the sake of simplicity, we used the aforementioned value in the present study. In addition, the observed results show that when droplets enter the irradiated region, the tilt of the top arc is smaller than $\beta$; that is, the bottom arc appears to be leaning to the right. By referring to the observed results, we simply assumed the angle of tilt of the upper arc as $0.5 \beta$. The contact-angle distribution on the contact line $\mathrm{C}$ in the figure is taken as $\theta(\mathrm{s})$, and the unit vector normal to line element 


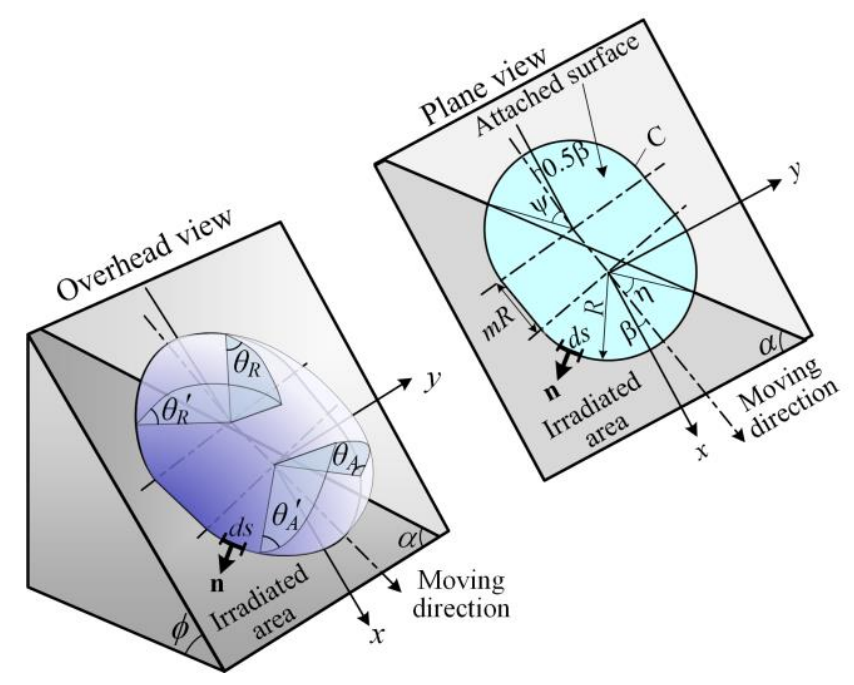

(This figure has been revised)

Fig. 4 Contact line of droplet entering into area of different contact angles $(\beta$ : incline of droplets' falling direction with respect to gravity; $R$ : radius of the top and bottom arcs of oval contact line. Other geometrical parameters are explained in the text)

$d s$ in the attached surface is represented by $\mathbf{n}$. Then, the integral of the surface tension and gravity acting upon the droplets along $\mathbf{C}$ can be used to obtain the vector of the force $\mathbf{F}$ applied to the droplets according to the following equation:

$\mathbf{F}=\oint_{C} \sigma \cos \theta \times \mathbf{n} d s+\rho g V \sin \phi \times \mathbf{e}_{\mathbf{x}}$

Here, $\mathbf{e}_{\mathbf{x}}$ represents the unit vector in the direction of gravity ( $x$ direction). As described in Section 2.2, in this experiment, observations were made at an inclination angle of the plate close to that for the condition under which the surface tension and gravity acting in the $x$ direction on the droplets are balanced. In this case, the maximum falling velocity of the droplets is at most $1 \mathrm{~mm} / \mathrm{s}$, and the influence of inertia and viscosity can be ignored compared with the gravitational force and surface tension.

As seen in Fig. 4, the boundary between the UV-irradiated and non-irradiated regions intersects the contact line $C$ at two points. The patterns of the intersection are categorized in five ways depending upon whether $C$ intersects a straight line or the top or bottom arc. For each situation, force $\mathbf{F}$ in Eq. (1) can be calculated, and the downward and transverse components $F_{x}$ and $F_{y}$, respectively, can be obtained. This result was used to calculate the falling angle $\beta$ of inclination for each droplet as $\tan \beta=\frac{F_{y}}{F_{x}}$.

The theoretical value of trajectory obtained from Eqs. (2) and (3) and the experimental values are 
compared in Fig. 5. The measured value of droplet position in the figure corresponds to the position of the center of gravity on the attached surface. Figure 5 shows the result for ethanol solution and water for the test plate with Pattern A when vibrations are not applied. As shown in the figure, the droplet movement is roughly approximated by the theoretical model used in this study.

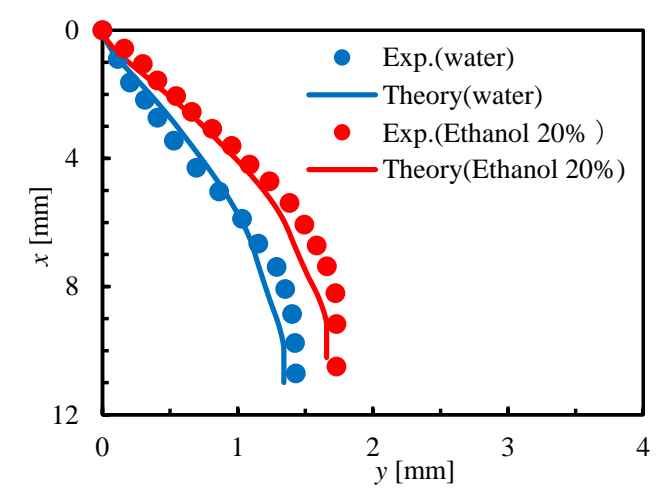

Fig. 5 Comparison of the experimental droplet trajectory with the theoretical model (Pattern A) ( $x$ and $y$ indicate the horizontal and gravitational directions, respectively)

\subsection{Evaluation of the direction of falling droplets}

As shown in Fig. 5, although the droplets in Pattern A eventually fall in the direction of gravity, they initially move in a fixed inclination angle from the direction of gravity over several millimeters. The maximum inclination angle in this case, $\beta_{\max }$, was $\sim 16^{\circ}$ for the ethanol solution and $\sim 10^{\circ}$ for water, as shown in Fig. 5. Here, $\beta_{\max }$ can be considered an index to determine the target inclination angle $\mu$ of patterns $\mathrm{B}$ or $\mathrm{C}$, as shown in Fig. 2.

We consider a representative situation in which the boundary line passes through the center of the attached surface of droplet (Fig. 4) to estimate the value of $\beta_{\max }$ theoretically. Then, $F_{x}$ and $F_{y}$ can be obtained as follows:

$$
\begin{aligned}
F_{x}= & \rho g V \sin \varphi+\sigma R\left[(1+\sin \eta) \cos \theta_{A}^{\prime}+(1+\sin \eta) \cos \theta_{A}-(1-\sin \psi) \cos \theta_{R}^{\prime}-(1+\sin \psi) \cos \theta_{R}\right], \\
F_{y}= & \sigma R\left[\cos \eta\left(\cos \theta_{A}-\cos \theta_{A}^{\prime}\right)+\cos \psi\left(\cos \theta_{R}-\cos \theta_{R}^{\prime}\right)+\left\{(1+\sin \eta) \cos \theta_{A}^{\prime}+(1-\sin \eta) \cos \theta_{A}\right\} \sin \beta\right. \\
& \left.-\left\{(1-\sin \psi) \cos \theta_{R}^{\prime}+(1+\sin \psi) \cos \theta_{R}\right\} \sin (0.5 \beta)\right]
\end{aligned}
$$

Here, $\eta$ and $\psi$ in Eqs. (4) and (5) are the angles shown in Fig. 4, and they satisfy the following relations: 
$\frac{\sin \eta}{m / 2+\cos \eta}=\tan \left(\frac{\pi}{4}-\beta\right), \quad \frac{\sin \psi}{m / 2+\cos \psi}=\tan \left(\frac{\pi}{4}-0.5 \beta\right)$

The values of $\beta$ calculated from Eqs. (3)-(6) matched the experimentally obtained values of $\beta_{\max }$ within a few degrees. Although the theoretical model proposed in this study assumes that $\beta$ is sufficiently small, i.e., $\beta \ll 1$ (Katoh et al. 2016), we can roughly evaluate $\beta_{\max }$ according to the contact angle by using Eq. (4) and (5). The first and second terms in the bracket on the right-hand side of Eq. (5) represent the force based on the difference between the contact angles in the irradiated and non-irradiated areas. On the other hand, the third and fourth terms multiplied by $\sin \beta$ and $\sin 0.5 \beta$, respectively, represent the contributions of the transversal components of the surface tension acting upon the top and bottom arcs of the contact line when the droplet is tilted by an angle $\beta$ or $0.5 \beta$ from the gravitational direction. As seen from Eq. (5), as each contact angle decreases, the first and second terms in the bracket increase, provided that the effect of irradiation $\left(\theta_{A}{ }^{\prime}-\theta_{A}\right)$ or $\left(\theta_{R}{ }^{\prime}-\theta_{R}\right)$ is not changed. On the other hand, the smaller the contact angle hysteresis $\left(\theta_{A}-\theta_{R}\right)$ or $\left(\theta_{A}{ }^{\prime}-\theta_{R}{ }^{\prime}\right)$, the greater is the sum of the third and fourth terms with the decrease (increase) in the advancing (receding) contact angle.

Using the contact angle for a water droplet when vibrations are not applied (Table 2) as the standard, the $\beta_{\max }$ values corresponding to the changes in the contact angles are obtained using Eqs. (3)-(6). We calculated $\beta_{\max }$ for three cases: case A and cases BA and BR. In case (A), we assumed that all $\theta_{A}, \theta_{R}$ and $\theta_{A}{ }^{\prime}, \theta_{R}{ }^{\prime}$ values were reduced for a fixed angle $\delta$. In case (B), we assumed that the contact angle hysteresis was reduced. Here, we considered two scenarios: case (BA), wherein the advancing contact angles, $\theta_{A}$ and $\theta_{A}{ }^{\prime}$, were reduced with $\delta$ and case (BR), wherein the receding contact angles, $\theta_{R}$ and $\theta_{R}{ }^{\prime}$, increased with $\delta$. The calculated results for water are shown in Fig. 6 . As shown in the figure, $\beta_{\max }$ for each case increases with $\delta$. A comparison of these three cases shows that the maximum $\beta_{\max }$ is obtained for case BA. If the difference between the contact angles in the irradiated and non-irradiated areas, $\Delta \theta$, is fixed in Eq. (5), the

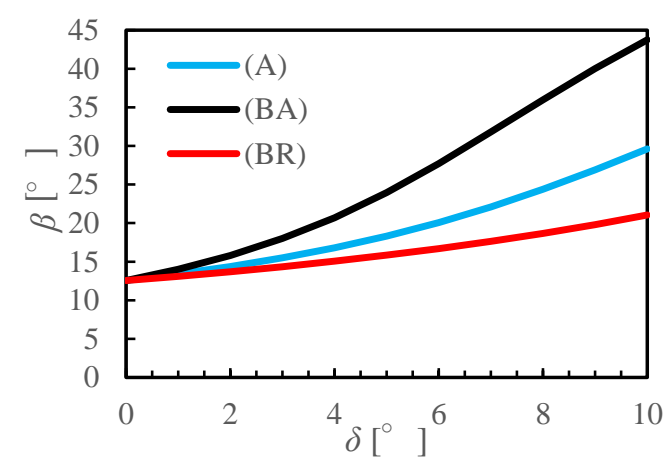

Fig. 6 Estimation of the inclination angle, $\beta$, from the gravitational direction (A: both advancing and receding contact angles are reduced by $\delta$. BA: only advancing contact angles are reduced by $\delta$. BR: only receding contact angles are reduced by $\delta$.) 
sum of the third and fourth terms in the bracket is greater than that of the first and second terms. Since sin $\beta>\sin 0.5 \beta$, force $F_{y}$ in Eq. (5) is increased to a greater extent when the third term is increased; that is, the advancing contact angle should be reduced. These results imply that the inclination angle $\beta$ of the falling droplets are effectively increased by applying ultrasonic vibrations that reduce the advancing contact angle.

\section{Experimental results and discussion}

The experimental results of droplet trajectory on the test plate with Pattern A for water and $20 \%$ ethanol solution are shown in Fig. 7; the results obtained with and without the application of ultrasonic vibration are plotted. The maximum inclination angle under vibration, $\beta_{\max }$, is $18^{\circ}$ in water and $19^{\circ}$ in the ethanol solution, showing an increase from their respective results of $10^{\circ}$ and $16^{\circ}$ when no vibration is applied. Moreover, in both test liquids, the droplets travel over longer distances at constant inclination angles on the plate under vibration. Under the conditions in this experiment, although the change in the contact angle, $\Delta \theta$, due to UV light is less owing to the application of ultrasonic vibration, as stated in section 2.2, $\beta_{\max }$ is larger than that obtained in the absence of vibrations because the advancing contact angle becomes smaller. When using the ethanol solution, the reduction in the advancing contact angle was smaller than that obtained with water, and the effect of ultrasonic vibration was not remarkable.

Referring to the results for Pattern A (Fig. 7), we determined the target of inclination angel $\mu$ for Patterns B and C (Fig. 2) corresponding to the condition of ultrasonic vibration. The target angle was set as $\mu=7^{\circ}$ and $\mu=12^{\circ}$, with and without ultrasonic vibration, respectively. The results of the measured droplet trajectory are shown in Fig. 8. Note that the following figures only shows the results for water because the effect of the ultrasonic vibrations was not significant for ethanol solution and the results of observation were almost similar to those obtained for water. Here, Figs. 8(a) and 8(b) represent the

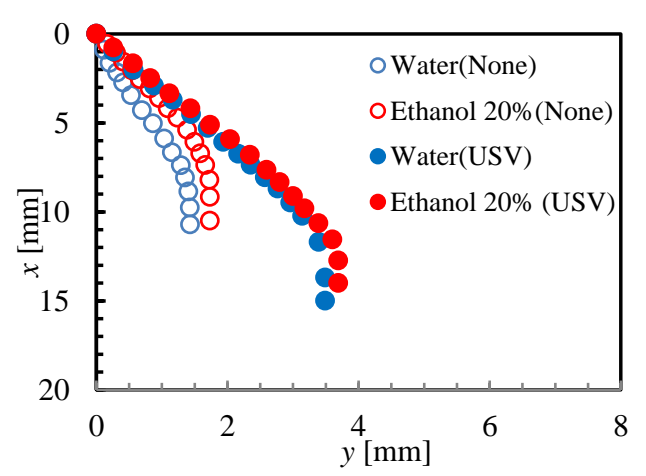

Fig. 7 Droplet trajectories with or without ultrasonic vibration for two types of test liquids ( $x$ and $y$ indicate the horizontal and gravitational directions, respectively) 
situations with and without ultrasonic vibration, respectively. For Patterns B and C, the droplets move along the set straight line at an inclination angle of $\mu=7^{\circ}$ [Fig. 8(a)]. On the contrary, although motion at the set angle $\mu=12^{\circ}$ is realized for Pattern $\mathrm{B}$, the droplets do not follow Pattern $\mathrm{C}$ and move at approximately $7^{\circ}$ [Fig. 8(b)]. Further, for Pattern C, the droplet has a smaller relative bend in the top and bottom arcs compared to those for Pattern A or B. In fact, the tilt angle of the top arc of the contact line is $\sim 0.7 \beta$, which is somewhat larger than $\sim 0.5 \beta$ for Patterns $\mathrm{A}$ and $\mathrm{B}$. This is attributed to the fact that in Patterns A and B, the tilt of the boundary of the wettability change is $45^{\circ}$; however, in Pattern $\mathrm{C}$, the majority of the boundary line is at $90^{\circ}$ (direction of gravity), thus reducing the relative bend in the top and bottom arcs. As the difference between the tilt angles of the top and bottom arcs decreases, the sum of the third and fourth terms in the bracket on the right-hand side of Eq. (5) too decreases. On the other hand, since the first term becomes smaller owing to the application of ultrasonic vibration, the transversal force, $F_{y}$, decreases. This suggests that the droplet could not reach the patterns with a large setting angle. The results obtained from the theoretical model are also shown in Fig. 8 for comparison. The experimental results are well approximated by the theoretical model even when the setting angle could not be achieved.

Considering the need for the practical control of droplet movement, we conducted experiments for a test plate with Pattern B for two setting angles, $\mu=7^{\circ}$ and $15^{\circ}$ (Fig. 9). The experimental results of the droplet trajectory are shown in Fig. 10. The droplets diverge from the direction of gravity immediately as they enter the saw-tooth pattern for $\mu=7^{\circ}$. Then, the droplet bends again immediately when it enters the region with the second target angle, i.e., $\mu=15^{\circ}$. Droplet motion at each inclination angle is shown in Fig. 10 ; it is also shown in an animation (Online Resource).

The changes in wettability are very small when the difference between the contact angles is $\sim 2^{\circ}$; however, the direction of motion can be controlled within $\sim 15^{\circ}$. The polymer used in this experiment has the characteristic that reversible-wettability-change patterning is realized under relatively simple UV

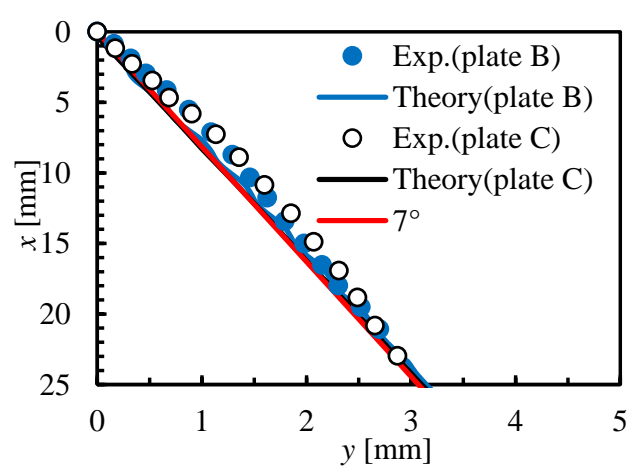

(a) Without vibration

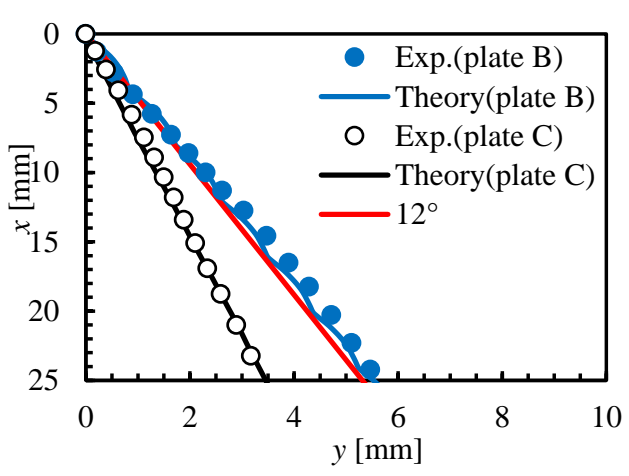

(b) With ultrasonic vibration

Fig. 8 Experimental results for the droplet trajectory for Patterns B and C (solid line indicates the theoretical results obtained from Eqs. (2) and (3) ) 
irradiation; however, in order to control the direction over a wider range, it is necessary to increase the change in contact angle, $\Delta \theta$; this is a topic for future research.

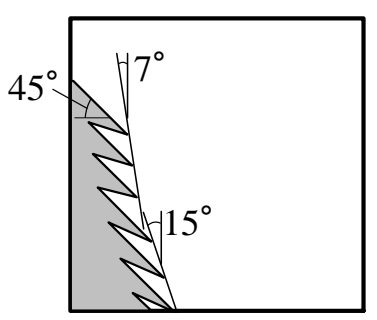

Fig. 9 Pattern B with two types of designated inclination angles of $7^{\circ}$ and $14^{\circ}$ (Gray area indicates the area with poor wettability resulted from irradiation of ultra-violet light)

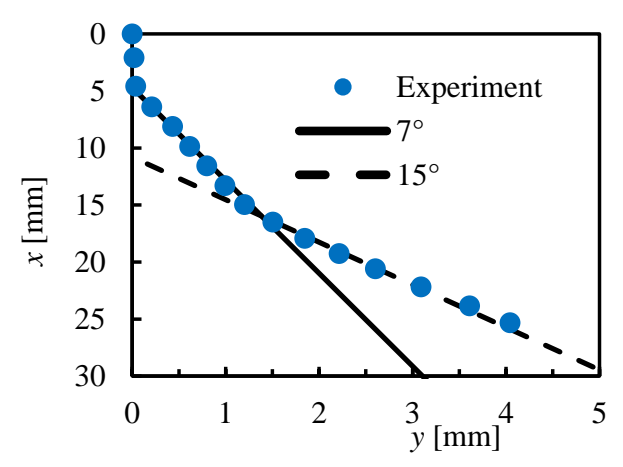

Fig. 10 Droplet trajectory on the test plate shown in Fig. 9 (Solid and broken lines indicate the target of droplet trajectory)

\section{Conclusion}

A method for controlling the direction in which droplets fall on an inclined plate is proposed. This method is based on the changes in wettability due to the cross-linking of polymers upon UV irradiation. Droplet behaviors on three types of test plates with different geometrical patterns of wettability change were observed. The main results can be summarized as follows:

(1) In the saw-tooth pattern proposed in this research, when the contact angle of the base plate changes, the change in the inclination angle $\beta$ of the droplet-falling direction with respect to gravity was considered based on the theoretical model in which the surface tension acting on the contact line and the gravitational force were considered. By decreasing the advancing contact angle of the base plate, $\beta$ was effectively increased.

(2) Ultrasonic vibration was applied to test plates, and the falling motion of the droplets was observed. For 
water, the contact angle between the UV-irradiated and non-irradiated areas decreased from $4^{\circ}$ without vibration to around $2^{\circ}$ with vibration; however, the advancing contact angle decreased by $\sim 8^{\circ}$ without vibration. Thus, $\beta$ increased considerably upon the application of vibration.

\section{Nomenclature}

\section{Roman symbols}

$B$ : Maximum width of a droplet [m]

$\boldsymbol{F}$ : Vector of the force applied to a droplet $[\mathrm{N}]$

g: Gravitational acceleration $\left[\mathrm{m} / \mathrm{s}^{2}\right]$

$m$ : Ratio of straight part of contact line to the radius of the arc-like contact line [-]

$R$ : Radius of arc-like contact line [m]

$V$ : Liquid volume $\left[\mathrm{m}^{3}\right]$

$x$ : Coordinate on the plate surface in the direction of gravity $[\mathrm{m}]$

$y$ : Coordinate on the plate surface in transverse direction [m]

\section{Greek symbols}

$\alpha\left(=45^{\circ}\right)$ : Oblique angle of the $\mathrm{UV}$-irradiated area in pattern $\mathrm{A}\left[^{\circ}\right]$

$\beta$ : Inclination angle of moving droplet $\left[{ }^{\circ}\right]$

$\delta$ : Variation in the contact angle $\left[^{\circ}\right]$

$\eta$ : Center angle of the arc-like contact line (advancing side, ref. Fig. 4) $\left[^{\circ}\right]$

$\theta$ : Contact angle $\left[{ }^{\circ}\right]$

$\mu$ : Target inclination angle of droplet movement $\left[{ }^{\circ}\right]$

$\rho$ : Liquid density $\left[\mathrm{kg} / \mathrm{m}^{3}\right]$ -

$\sigma$ : Surface tension $[\mathrm{N} / \mathrm{m}]$

$\phi$ : Inclination angle of the test plate $\left[{ }^{\circ}\right]$

$\psi$ : Center angle of the arc giving the receding contact angle (receding side, ref. Fig. 4 ) $\left[{ }^{\circ}\right]$

\section{Superscripts}

': UV-irradiated

\section{Subscripts}
A: Advancing
R: Receding
$x$ : Gravity direction on the plate surface
$y$ : Transverse direction on the plate surface

\section{References}


Abdelgawad M, Park P, Wheeler R (2009) Optimization of device geometry in single-plate digital microfluidics. J Applied Phys 105: 094506. https://doi.org/10.1063/1.3117216

Abgrall P and Gué AM (2007) Lab-on-chip technologies: making a microfluidic network and coupling it into a complete microsystem-a review. J. Micromech Microeng 17: R15-R49. https://doi.org/10.1088/0960-1317/17/5/R01

Agapov RL, Boreyko JB, Briggs DP, Srijanto BR, Retterer, ST, Collier CP, Lavrik NV (2014) Length scale selects directionality of droplets on vibrating pillar ratchet. Adv Mater Interfaces 1: 1400337. https://doi.org/10.1002/admi.201400337

Bhaumik SK, Das S, Chakraborty S, Das Gupta S (2014) Droplet Transport through dielectrophoretic actuation using line electrode. Microfluid Nanofluidics 16: 597-603. https://doi.org/10.1007/s10404-013-1242-5

Chen JZ, Troian SM, Darhuber AA, Wagner S (2005) Effect of contact angle hysteresis on thermocapillary droplet actuation. J Appl Phys 97: 014906. https://doi.org/10.1063/1.1819979

Darhuber AA, Troian SM (2005) Principles of microfluidic actuation by modulation of surface stresses. Annu Rev Fluid Mech 37: 425-455. https://doi.org/10.1146/annurev.fluid.36.050802.122052

Datta S, Das AK, Das PK (2015) Uphill movement of sessile droplets by electrostatic actuation. Langmuir 31: 10190-10197. https://doi.org/10.1021/acs.langmuir.5b02184

Dong Y, Holmes HR, Böhringer KF (2017) Converting vertical vibration of anisotropic ratchet conveyors into horizontal droplet motion. Langmuir 33: 10745-10752. https://doi.org/10.1021/acs.langmuir.7b02504

Fobel R, Fobel C, Wheeler AR (2013) Dropbot: An open-source digital Microfluidic control system with precise control of electrostatic driving force and instantaneous drop velocity measurement. Appl Phys Lett 102: 193513. https://doi.org/10.1063/1.4807118

Gupta S, Ramesh K, Ahmed S, Kakkar V (2016) Lab-on-chip technology: a review on design trends and future scope in biomedical applications. International Journal of Bio-Science and Bio-Technology 5: 311-322. https://doi.org/10.14257/ijbsbt.2016.8.5.28

Higashine M, Katoh K, Wakimoto T, Azuma T (2008) Profiles of liquid droplets on solid plates in gravitational and centrifugal fields. J JSEM 8(Special Issue): 49-54. https://doi.org/10.11395/jjsem.8.s49

Hutama TJ, Oleschuk RD (2017) Magnetically manipulated droplet splitting on a 3D-printed device to carry out a complexometric assay. Lab Chip 17: 2640-2649. https://doi.org/10.1039/C7LC00629B

Jain V, Devarasetty V, Patrikar R (2017) Effect of electrode geometry on droplet velocity in open EWOD based device for digital microfluidics applications. $\mathrm{J}$ Electrostatics 87: 11-18. https://doi.org/10.1016/j.elstat.2017.02.006

Karapetsas G, Chamakos NT, Papathanasiou AG (2017) Thermocapillary droplet actuation: effect of solid structure and wettability. Langmuir 33: 10838-10850. https://doi.org/10.1021/acs.langmuir.7b02762 
Katoh K, Higashine M, Nakamoto N and Azuma T (2006) On the sliding down of liquid drops on inclined plates (1st report, Critical inclination angle of plates). Trans Jpn Soc Mech Eng Ser B 72: 1287-1294. https://doi.org/10.1299/kikaib.72.1287

Katoh K, Wakimoto T, Masuda R (2010) A new method to actuate a droplet on a plate by use of laser and ultrasonic oscillation. Trans Jpn Soc Mech Eng Ser B 76: 2135-2142. https://doi.org/10.1299/kikaib.76.772_2135

Katoh K, Tamura H, Sato E, Wakimoto T (2016) Control of droplet movement on an inclined wall by difference of wettability. Jap J Multiphase Flow 29: 451-459. https://doi.org/10.3811/jjmf.29.451

Lahoon S, Rio OI, Cheng P, Neumann AW (Edited by Neumann AW Spelt JK) (1996) Axisymmetric drop shape analysis (ADSA). Applied Surface Thermodynamics Surfactant Sci Ser 63: 441-508 CRC Press.

Lee JL, Lee S, Kang KH (2012) Droplet jumping by electrowetting and its application to the three-dimensional digital microfluidics. Appl Phys Lett 100: 081604. https://doi.org/10.1063/1.3688487

Li W, Lynch V, Thompson H, Fox MA (1997) Self-assembled monolayers of 7-(10-Thiodecoxy)coumarin on gold: synthesis, characterization, and photodimerization. J Am Chem Soc 119: 7211-7217. https://doi.org/10.1021/ja970633 m

Lu HW, Glasner K, Bertozzi AL, Kim CJ (2007) A diffuse interface model for electrowetting drops in a Hele-Shaw cell. J Fluid Mech 590: 411-435. https://doi.org/10.1017/S0022112007008154

Morrison H, Curtis H, McDowell T (1966) Solvent effects on the photodimerization of coumarin ${ }^{1}$. J Am Chem Soc 88: 5415-5419. https://doi.org/10.1021/ja00975a009

Nahar MM, Nikapitiya JB, You SM, Moon H (2016) Droplet velocity in an electrowetting on dielectric digital microfluidic device. Micromachines 7: 71. https://doi.org/10.3390/mi7040071

Obi, M, Morino S, and Ichimura K (1999) Factors affecting photoalignment of liquid crystals induced by polymethacrylates with coumarin side chains. Chem Mater 11: 656-664. https://doi.org/10.1021/cm980533v

Pratap V, Moumen N, Subramanian RS (2008) Thermocapillary motion of a liquid drop on a horizontal solid surface. Langmuir 24: 5185-5193. https://doi.org/10.1021/la7036839

Sato E, Nagai S, Matsumoto A (2012) Reversible volume changes of polymer thin films and their application to wettability control. 8th Coatings Science International Conference Book of Abstracts: 83-86.

Sato E, Nagai S, Matsumoto A (2013) Reversible thickness control of polymer thin films containing photoreactive coumarin derivative units. Prog Org Coat 76: 1747-1751. https://doi.org/10.1016/j.porgcoat.2013.05.010

Shastry A, Case MJ, Böhringer KF (2006) Directing droplets using microstructured surfaces. Langmuir 22: 6161-6167. https://doi.org/10.1021/la0601657 
Suzuki K, Homma H, Murayama T, Fukuda S, Takanobu H, Miura H (2010) Electrowetting-based actuation of liquid droplets for micro transportation systems. J Adv Mech Des Syst 4: 365-372. https://doi.org/10.1299/jamdsm.4.365

Wakimoto T, Sato Y, Katoh K (2013) A new method to actuate a droplet on a plate by use of laser irradiation to improve wettability. J JSEM 13: 19-26. https://doi.org/10.11395/jjsem.13.19

Volpe CD, Maniglio D, Morra M, Siboni S (2002) The determination of a 'stable-equilibrium' contact angle on heterogeneous and rough surfaces, Colloids Surf A Physicochem Eng Asp 206: 47-67. https://doi.org/10.1016/S0927-7757(02)00072-9

Xi HD, Zheng H, Guo W, Gañán-Calvo AM, Ai Y, Tsao CW, Zhou J, Li W, Huang Y, Nguyenh NT, Tan SH (2017) Active droplet sorting in microfluidics: a review. Lab Chip 17: 751-771. https://doi.org/10.1039/C6LC01435F

Yeo LY, Friend JR (2014) Surface acoustic wave microfluidics. Annu Rev Fluid Mech 46: 379-406. https://doi.org/10.1146/annurev-fluid-010313-141418

Zhang Y, Nguyen NT (2017) Magnetic digital microfluidics - a review. Lab Chip 17: 994-1008. https://doi.org/10.1039/C7LC00025A

Zhao Y, Liu F, Chen CH (2011) Thermocapillary actuation of binary drops on solid surfaces. Appl Phys Lett 99: 104101. https://doi.org/10.1063/1.3632041 